THURSDAY，DECEMBER 27, 1883

\section{VORTEX RINGS}

The Motion of Vortex Rings. By J. J. Thomson. (London: Macmillan and Co., 1883)

BOTH as regards the interest of the subject and the $B$ treatment it has received at the hands of the author we do not doubt that the essay before us is destined to take a foremost place amongst the essays which have been called forth, or at all events distinguished, by the Adams Prize.

The fact that these essays are upon set subjects precludes the possibility of the prize being awarded for a distinctly original conception. It is almost a necessity that the subjects chosen should involve the extension of some mathematical investigation which has already been carried a certain length.

The subject of the present essay is distinctly of this class; it involves an extension of the investigation of the theory of vortex motion in an ideal fluid, founded by Helmholtz and continued chiefly by Sir William Thomson.

At the time Helmholtz conceived the fundamental principle, ideal hydrodynamics had no other interest, besides its mathematical interest, than it derived from the somewhat casual explanations it affords of the phenomena met with in the motion of actual fluids. Helmholtz's investigation had some relation to the observed phenomena of actual vortices, particularly to the phenomena of smoke rings, of which it afforded a general explanation. But between the fundamental equations which Helmboltz gave and their application to an actual vortex ring certain integrations were necessary, and these integrations presented mathematical difficulties. If we consider the line of smoke which forms the ring as indicating the portion of air in which vortex motion exists, we may say that the difficulties of integration at which Helmholtz stopped arise from the thickness of this line of smoke, or, calling this the circular core of the ring, from the finite area of the section of this core. Helmholtz contented himself with applying his theory to an indefinitely thin core; and the fact that the results of a theory based on a frictionless fluid would only have an imperfect relation to the motions of viscous fluids, together with the fact that such rings, although they may be produced by artificial apparatus, are short-lived, and have no existence in the general motion of fluids, offered but little inducement for farther prosecution of the subject. The case however was altered when it was conceived by Sir William Thomson that the atoms of matter may be such rings moving in a perfect universal fluid. Smoke rings, although their behaviour seems to have suggested the idea, could not, owing to the viscosity of the air, by any means be made to afford an experimental verification of the capabilities of such an bypothesis. The only way was to integrate Helmholtz's equations, and thus arrive at the theoretical behaviour of such rings. Unfortunately the mathematical difficulties are such that there is little hope of obtaining a complete theory of vortex rings having cores of any finite area. Sir William VOL. XXIX.-NO. 739
Thomson, however, started an approximate theory as a step towards this; he succeeded in approximately integrating the equations for rings the cores of which had sections finite but small compared with the openings of the rings, and with such rings it appears that his theory can be tested as regards matter in the gaseous state.

To do this, however, it is necessary to do more than work out the theory of a single circular ring baving a core of circular section. The phenomena of gases depend on the internal vibration of the atoms and on the influence which they exert on each other by collisions or otherwise. It was necessary therefore to obtain the theory of the vibrations of these rings, also of the effect of what may be called collisions.

Sir William Thomson took many steps towards the theory of vibrations. But the theory of collisions was left for Mr. J. J. Thomson.

Mr. Thomson has not, however, confined his attention to the point set for the prize, but, starting from the foundation laid by Helmholtz, has recast the theory to his own method.

Having deduced general expressions for the momentum, moment of momentum, and energy in a mass of fluid in which there is vortex motion, which expressions are better adapted for his purpose than any previously obtained; he proceeds to the theory of a solitary vortex ring subject to the same limitation as that treated by Sir William Thomson, i.e. the diameter of the core small compared with the opening of the ring, but of more general shape, in that it may have any small deviation from the circular form. He obtains results which, where they correspond, agree very approximately with those previously obtained by Sir William Thomson.

The author then proceeds to the immediate subject of the essay - the action upon each other of two rings.

In dealing with this subject he introduces another important limitation, i.e. that the rings shall not approach each other by a distance which is large compared with the openings of the rings.

With this limitation, by means of a very powerful piece of mathematical work, the theory of the mutual action of such rings is deduced, both as regards mean motion and vibration; and he has thus carried the theory of vortex atoms to such a stage that in certain general respects it can be applied to the theory of gases.

The essay, however, does not end here, for, although outside the set subject, the author proceeds to consider the theory of "linked rings." This term does not seem well chosen, for it conveys the idea of rings linked as in a chain, whereas what it is used to express is a ring of which the core is compounded of several separate cores wrapped in a spiral manner round each other like a ring composed of twisted wire.

In the treatment of this branch of his subject he has been no less successful than in the earlier parts.

From the general scheme of his essay it is clear that the author has had in his mind as a general object the verification of the vortex atom theory; and although he avowedly refrains from going at length into such a vortex atom theory of gases as might be built upon his work, he adds a chapter at the end in which be discusses certain results of his work, which may be applied without further calculation to the vortex atom theory of gases. 
It is this chapter which will excite the most general interest, for although the fact of this still very incomplete theory being found consistent with observed gaseous phenomena would not afford a crucial test of its fitness to explain the phenomena of solids and liquids, still its failure to explain the phenomena of gases would appear to be crucial as regards its unfitness as an atomic theory.

The fair and cautious spirit in which Mr. Thomson discusses his results cannot be too much admired, although we may not be quite able to realise the truth of his reasoning.

The most general and important phenomenon of gases is that sometimes called Boyle's law-that the product of the volume and pressure of any fixed weight of gas varies directly as the amount of heat, i.e. kinetic energy, in a gas.

Accordingly $\mathrm{Mr}$. Thomson calculates the product of the pressure and volume which would result in the case of a vortex atom gas. This he finds equal to two terms. one being the kinetic energy multiplied by a constant, the other a certain quantity which involves the squares of the velocity of the medium at the boundary surface. To fit Boyle's law this second term must vanish or nearly so. Mr. Thomson argues that it does so vanish, because the surface being at rest the velocity of the fluid at it must be small. This argument we entirely fail to follow, possibly owing to some misapprehension on our part; but it seems to us that a vortex being near a solid surface is no reason for supposing the tangential velocity of the fluid small, while if the gas consists of vortex atoms so must the solid surface, and there is nothing to show that the mean square of the velocity within the solid and at its surface will be less than in the gas.

Passing on from Boyle's law, with the explanation of which he is satisfied, the author next turns to the phenomena depending on the velocity of the gaseous molecules. As this seams to us the most interesting part of the dis. cussion, we quote the passage in full :-

"According to the vortex atom theory, as the temperature rises and the energy increases the mean radius of the vortex rings will increase, but when the radius of a vortex ring is increased its velocity is diminished, and thus the mean velocity of the molecules decreases as the temperature increases; thus it differs from the ordinary kinetic theory, where the mean velocity and the temperature increase together. It ought to be remarked, however, that though in the vortex atom theory the mean velocity decreases as the temperature increases, yet the mean momentum increases with the temperature.

"The difference between the effects produced by a rise in temperature on the mean velocity of the molecules will probably furnish a crucial experiment between the vortex atom theory and the ordinary kinetic theory of gases, since all the laws connecting the phenomena of diffusion with the temperature can hardly be the same for the two theories. In fact. if we accept Maxwell's reasoning about the phenomenon called 'thermal effusion' we can see at once an experiment which would decide between the two theories.

"The phenomenon is this, if we have a porous diaphragm immersed in a gas, and the gas at the two sides of the diaphragm at different temperatures, then when things have got into a steady state the pressures on the two sides of the diaphragm will be different, and Maxwell, in his paper 'On Stresses in Rarefied Gases' (Phil. Trans. I 879 , part i. p. 255), gives the following reasoning to prove that, according to the ordinary theory of gases, the pressures on the two sides are proportional to the square root of the absolute temperatures of the sides. He says:--

"When the diameter of the hole and the thickness of the plate are both small compared with the length of the free path of the molecule, then, as Sir W. Thomson has shown, any molecule which comes up to the hole on either side will be in very little danger of encountering another molecule before it has got fairly through to the other side.

"Hence the flow of gas in either direction through the hole will take place very nearly in the same manner as if there had been a vacuum on the other side of the hole, and this whether the gas on the other side of the hole is of the same or of a different kind.

" "If the gas on the two sides of the plate is of the same kind but at different temperatures, a phenomenon will take place which we may call thermal effusion. The velocity of the molecules is proportional to the square root of the absolute temperature, and the quantity which passes out through the hole is proportional to this velocity and to the density. Hence, on whichever side the product of the density into the square root of the temperature is greatest, more molecules will pass from that side than from the other through the hole, and this will go on till this product is equal on both sides of the hole. Hence the condition of equilibrium is that the density must be inversely as the square root of the temperature, and since the pressure is as the product of the density into the temperature, the pressure will be directly proportional to the square root of the absolute temperature.

"If we were to apply the same reasoning to the vortex atom theory, we should no longer have the velocity proportional to the square root of the absolute temperature, but to some inverse power of it, and the above reasoning would show that if $p$ and $p^{\prime}$ be the pressures, $t$ and $t^{\prime}$ the temperatures on the two sides of the plate, $p / p^{\prime}=\left(t / t^{\prime}\right)^{m}$, where $m$ is a quantity greater than unity. Thus accurate investigations of the phenomenon of thermal effusion would enable us to decide between the vortex atom and the ordinary kinetic theory of gases. These experiments would, however, be difficult to make accurately, as we should have to work with such low pressures to get the mean path of the molecules long enough that the pressure of the mercury vapour in the air-pump used to rarefy the gas might be supposed sensibly to affect the results. In the theoretical investigation, too, the effects of the bounding surface in modifying the motion of the gas seem to have scarcely been taken sufficiently into account to make the experiment of the crucial test of a theory ; and it is probable that the theory of the diffusion and viscosity of the gases worked out from the laws of action of two vortex rings on each other, given in Part II. of this essay, would lead to results which would decide more easily and more clearly between the two theories.

"The preceding reasoning holds only for a monatomic gas which can only increase its energy by increasing the mean radius of its vortex atoms; if, however, the gas be diatomic, the energy will be increased if the shortest distance between the central lines of the vortex cores of the two atoms be diminished, and if the radius of the vortex atom is unaltered the velocity of translation of the molecule will be increased as well as the energy; thus for a diatomic molecule we cannot say that an increase in the energy or a rise in the temperature of the gas would necessarily be accompanied by a diminution in the mean velocity of its molecules."

With the argument here used we have no fault to find, but it does seem to us that the author bas fallen into some confusion between the experimental phenomenon of thermal transpiration through porous plugs and the theoretical idea of "thermal effusion." It has probably escaped Mr. Thomson, but the experiment he suggests 
was included in the general investigation, made by the writer of the present review, ${ }^{1}$ by which the phenomenon of thermal transpiration was discovered, and although it still appears that these are the only experiments on this subject, yet they conclusively prove that the difference of the pressure on the two sides of the plate is proportional to the square roots of the absolute temperatures. So far then it would seem that the crucial experiment has been made and that the verdict is against the vortex atom theory; but this is not so, for, although the experiment Mr. Thomson suggests has been made, it is definitely and experimentally shown in the same investigation that the action of the porous plug is entirely different from that which Maxwell calls thermal effusion, being due entirely to the tangential action of the walls of the passages, and further this tangential action is in strict accordance with the present dynamical theory of gases. This experiment with the porous plug, then, affords no test whatever in the way suggested by Mr. Thomson. Mr. Thomson has, we think, been unfortunate in bis choice of tests; and we would suggest the velocity of sound as affording a crucial test for which the experimental work is already done. It appears to be an almost obvious deduction from the vortex atom theory that the velocity of sound must be limited by the mean velocity of the vortex atoms; and since Mr. Thomson has shown that this mean velocity diminishes with the temperature, while experimentally it is found that the velocity of sound increases as the square root of the temperature, it appears that the verdict must be against the vortex atom theory. However the vortex atoms are very slippery things, and we should like to hear Mr. Thomson's opinion before adopting one of our own.

Besides discussing the theory of gases, Mr. Thomson goes somewhat fully into a vortex atom theory of chemical combinations; in this he raises many points which will doubtless be of great interest should the hypothesis survive the crucial test by the theory of gases which this essay now for the first time renders possible.

Of the mathematical interest of the essay we can only say that to those who can appreciate it this will be found to be very great.

OSBORNE REYNOLDS

\section{OUR BOOK SHELF}

Krystallographische Untersuchungen an homologen und isomeren Reiken. Von Dr. A. Brezina. I. Theil. Methoden. (Wien, 1884.)

THIS very useful volume forms an introduction to the author's crystallographic investigations which earned the prize of the Vienna Academy. It deals exclusiveiy with the principles and the methods employed in those investigations, and constitutes a complete storehouse of the formula required in the study of crystals, and of the best means of applying those formulæ. The following subjects are successively treated: the optical principles involved in the goniometer; the practical use of the instrument, and the errors to which it is liable; the criticism of probable errors of observation; stereographic projection; all possible cases of trigonometrical calculation, including the methord of least squares; and a slight sketch of the use of the polarising apparatus.

An important feature of the book is the illustration of methods by the actual measurement of seven crystals of a triclinic substance. The readings of the goniometer scale are first given, and from these the reader is led

1 "Certain Dimensional Properties of Matter in the Gaseous State," Phil. Trans. I879, Part II. through the entire series of processes: stereographic projection, assignment of indices, calculation of clements, and recalculation of angles, each given in its place as an example of the principles and formulæ enployed. This practical illustration is a far more effectual means of recommending the methods to the reader than mere verbal description.

It will probably be found that these methods of calculation are the most valuable part of the book; they are so systematically arranged and tabulated that the various steps may be distinguished at a glance, and any numerical error must be detected at once, while much labour is saved by the methodical order in which the operations are conducted.

It is to be presumed that the laborious process of calculating the angle between each pair of faces from the elements by means of the general formula is given as an exercise in the method of least squares rather than as an example of the course to be actually adopted in any but rare cases.

One subject, however, of some importance is barely touched upon; namely, the criticism of images obtained from crystal faces on the goniorncter, and their interpietation. Both in the descriptive paragraphs and in the above-mentioned illustration, all measurements of the same angle upon different crystals are assumed to be equally good, so that their arithmetic mean is adopted as the observed value, whereas the difficulties presented by multiple images seem to deserve treatment in a book which deals so exhaustively with the practical side of the subject. It is to be regretted also that the discussion of optical properties and measurement has been almost crowded out of the work.

H. A. M.

\section{LETTERS TO THE EDITOR}

[The Editor does not hold himself responsible for opinions expressed by his correspondents. Neither can he undertake to return, or to correspond with the writers of, rejected manuscripts. No notice is taken of anonymous communications.

[The Editor urgently requests correspondents to keep their letters as short as possible. The pressure on his space is so grat that it is impossible otherwise to insure the appearance even of communications containing interesting and novel facts.]

\section{The Remarkable Sunsets}

SPEAIIING of Virginia City, the great silver mining centre of Nevada State, I said, in "An Engineer's Holiday," that it "lies among the foothills of the Sierra, at an elevation of 6200 feet, on the eastern face of Mount Davidson . . . surrounded by innumerable interlocked mountains, conical in sutline, red-brown in colour, and perfectly bare of all vegetation. These stretch, as far as the eye can reach, to where the snowy tops of the Humboldt peaks stand against the sky, and the terrible sterility of the scene is enhanced rather than relieved by the thin meanderings of the Carson River, whose course is marked by a narrow green line. This is the only sign of water visible in the arid panorama, whose bare, red cones are steeped all day in dusthaze, and lighted for a few minutes at sunset by an 'Alpen. glow' which dyes the countless peaks in as countless gradations of rosy light."

It certainly did not occur to me, when I wrote the above three years ago, that the finer and higher particles of the drsthaze which obscures the dry air of the American desert may have been conceroed in producing the splendid sunset effects which I witnessed at Virginia City; but this, after our recent experiences, seems very probable. D. PIDGEON

Holmwood, Putney Hill, December 22

I HAVE received a letter, dated December 5 , from Mr. Joseph Moore, of New Garden, North Carolina, U.S.A., in which he informs me that "the phenomena at both sunset and sunrise have been unusual in more than a dozen instances here during the autumn. Only the night before last we had an extraordinary sunset. The sky bore all the tints of which you speak, but $I$ do 